\title{
Parry-Romberg syndrome associated with en coup de sabre in a patient from South Sudan - a rare entity from East Africa: a case report
}

\author{
Jimmy Girgis William Abdelnour ${ }^{1 *}$ (D), Youeil Girgis William Abdelnour ${ }^{1}$, Rose-Mery Amin Boushra Kerollos ${ }^{2}$ and \\ Ziryab Imad Taha Mahmoud ${ }^{3}$
}

\begin{abstract}
Background: Parry-Romberg syndrome, also known as progressive hemifacial atrophy, is a rare degenerative disorder with numerous distinctive clinical presentations. It is usually slowly progressive, occurring more in females, and affects primarily one side of the face; it causes unilateral atrophy and loss of skin, subcutaneous tissue, muscles, and bones, and can even extend to oral structures. Other involvements that can occur are ocular and neurological; however, it is frequently associated with linear scleroderma, known as en coup de sabre. The etiology of the disorder is unknown, although some consider it a variant of morphea (localized scleroderma) and others proposed a malfunction of the sympathetic system as a cause. Imaging studies can support diagnosis and reveal the extent of the disease. Moreover, with the wide systemic involvement in such a condition, a multidisciplinary approach is crucial.

Case presentation: A 35-year-old Dinka woman presented with left hemifacial atrophy associated with left-sided body hemihypoesthesia and glaucoma with overlapping linear scleroderma "en coup de sabre" for 5 years.

Conclusions: Parry-Romberg syndrome is a very rare entity causing progressive hemifacial atrophy that could also be associated with linear scleroderma. It has devastating outcomes due to its various systemic involvements; therefore, a multidisciplinary approach is required together with further studies to be performed in order to identify the key etiology and construct a clear guideline for management.
\end{abstract}

Keywords: Parry-Romberg disease, Linear scleroderma, En coup de sabre, Progressive hemifacial atrophy

\section{Background}

Parry-Romberg syndrome (PRS), also known as progressive hemifacial atrophy, was originally described by Caleb Hillier Parry in 1815, then it was described with further details by Moritz Heinrich Romberg in 1846 and in 1871 Eulenberg established the term "progressive facial hemiatrophy" [1-3]. It is a developmental craniofacial disorder of unknown etiology characterized by a slowly progressive unilateral facial atrophy and is associated with different systemic manifestations. In particular, it is associated with maxillofacial manifestations (wasting of masticatory muscles, delayed ipsilateral tooth eruption, unilateral tongue atrophy,

* Correspondence: jimgw20@gmail.com

${ }^{1}$ Abu-Rof Clinic, Omdurman, Sudan

Full list of author information is available at the end of the article jaw hypoplasia), and neurological (trigeminal neuralgia, migraine, seizures) and ophthalmologic abnormalities (enophthalmos, glaucoma, endothelial precipitates, Horner's syndrome, ophthalmoplegia) [4-9]. Moreover, it is frequently associated with linear scleroderma and referred to as en coup de sabre $[10,11]$.

Onset of the disease is in childhood, typically during the first two decades of life, although later onset has previously been described. Usually, the atrophy progresses slowly for several years and afterward it becomes stable with females being affected more than males [11, 12].

Almost two centuries after this disease was defined, the etiology has still not been determined. Autoimmune disease and other causes like metabolic disorders and trauma have been 


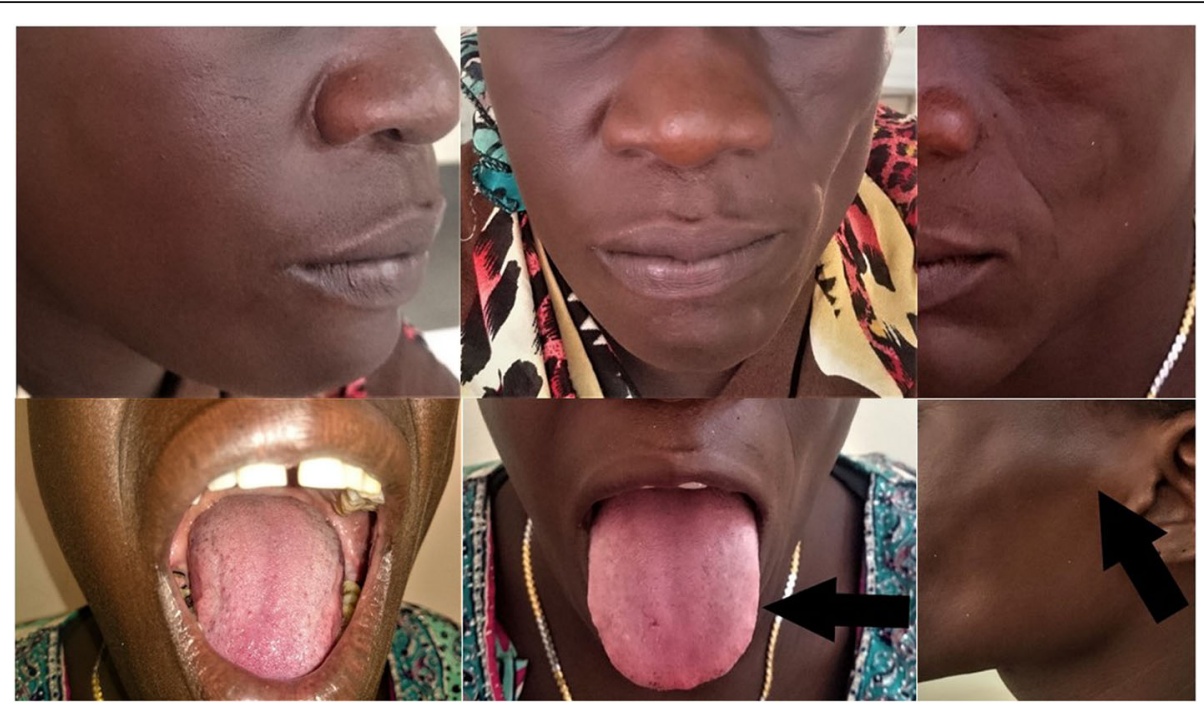

Fig. 1 Photographs of the patient from different views showing the obvious atrophy involving the left side of her face, minimal tongue atrophy (arrow), clear demarcation of en coup de sabre (arrow), and the intraoral dental involvement

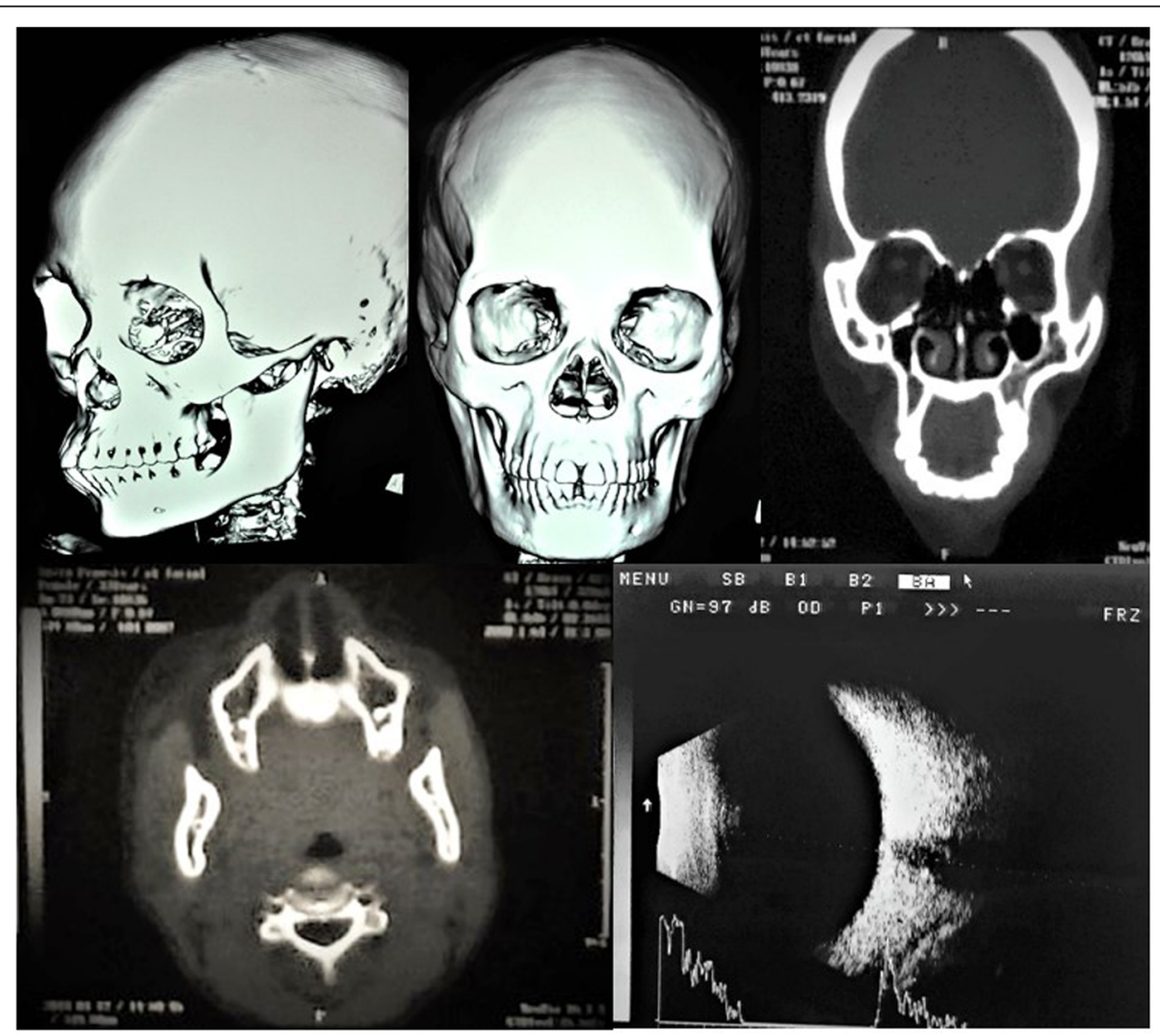

Fig. 2 Brightness scan (ophthalmic ultrasonography) of the left eye showing no abnormalities; three-dimensional computed tomography of facial bones showing mild left zygomatic bone involvement and computed tomography showing decreased size of orbital bones on left side together with atrophy of facial subcutaneous and masseter muscle on the affected side 
Table 1 Lab Test Results

\begin{tabular}{|c|c|c|c|c|c|}
\hline \multicolumn{6}{|c|}{ Laboratory Results } \\
\hline \multirow[t]{12}{*}{$\mathrm{CBC}$} & $\mathrm{Hb}$ & 10.6 & ESR & \multicolumn{2}{|l|}{$10 \mathrm{~mm} / \mathrm{hour}$} \\
\hline & $P C V$ & 32.1 & Vitamin $\mathrm{B}_{12}$ & \multicolumn{2}{|c|}{$412 \mathrm{pg} / \mathrm{mL}(221-950)$} \\
\hline & $R B C S$ & 3.99 & Rh. factor & \multicolumn{2}{|c|}{$>8 \mathrm{IU} / \mathrm{mL}$ Positive } \\
\hline & MCV & 80.5 & Anti-CCP & $9.7 \mathrm{AU} / \mathrm{mL}$ & Negative \\
\hline & $\mathrm{MCH}$ & 26.6 & & & \\
\hline & MCHC & 33 & & $S M$ & Negative \\
\hline & TWBCS & 3900 & & $S S-A$ & Negative \\
\hline & $N$ & $54 \%$ & & Ro52 & Negative \\
\hline & L & $37 \%$ & & $S S-B$ & Negative \\
\hline & $M$ & $8 \%$ & & SCL 70 & Negative \\
\hline & $E$ & $1 \%$ & ANA profile & JO-1 & Negative \\
\hline & $P L T$ & 265,000 & & DsDNA & Negative \\
\hline \multirow[t]{6}{*}{ RFT } & Urea & 24 & & Nucleosomes & Negative \\
\hline & Creatinine & 0.7 & & Histones & Negative \\
\hline & $\mathrm{Na}^{+}$ & 132 & & Rib p-protein & Negative \\
\hline & $K^{+}$ & 3.6 & & AMA M2 & Negative \\
\hline & $\mathrm{Ca}^{2+}$ & 9.9 & & PCNA & Negative \\
\hline & $\mathrm{PO}^{3+}$ & 3.4 & & PM-SCL & Negative \\
\hline \multirow[t]{10}{*}{ LFT } & T. protein & 7.3 & & CENPB & Negative \\
\hline & & & & Nrnp/SM & Negative \\
\hline & S. albumin & 4.5 & & & \\
\hline & Globulin & 2.8 & & & \\
\hline & T. bilirubin & 0.52 & & & \\
\hline & D. bilirubin & 0.3 & & & \\
\hline & 1. bilirubin & 0.22 & & & \\
\hline & $A L T$ & 16 & & & \\
\hline & AST & 20 & & & \\
\hline & $A L P$ & 72 & & & \\
\hline
\end{tabular}

ALP alkaline phosphatase, $A L T$ alanine aminotransferase, $A M A M 2$ antimitochondrial M2 antibody, AST aspartate aminotransferase, $A N A$ antinuclear antibody, CBC complete blood count, CCP cyclic citrullinated peptide, CENPB centromere protein $B, D$. direct, $D$ s double-stranded, $E$ erythrocyte, $E S R$ erythrocyte sedimentation rate, $H b$ hemoglobin, I. indirect, $L$ leukocyte, LFT liver function test, $\mathrm{M}$ monocyte, $\mathrm{MCH}$ mean corpuscular hemoglobin, $\mathrm{MCHC}$ mean corpuscular hemoglobin concentration, $M C V$ mean corpuscular volume, $N$ neutrophil, $P C N A$ proliferating cell nuclear antigen, $P C V$ packed cell volume, PLT platelet, $P M-S C L$ polymyositis/scleroderma (exosome) autoantigen, $R B C$ red blood cells, RFT renal function test, $R h$. rhesus, Rib ribosomal, $S$. serum, SS- $A$ Sjögren syndrome antigen $A$ or Ro, SS-B Sjögren syndrome antigen $B$ or $L a, T$. total, TWBC total white blood cell

postulated; sometimes, familial cases with autosomal dominant inheritance have been seen.

Concerning the treatment, there is no cure although reconstructive surgery with immunosuppressive drugs have been used and showed considerable results [10, 13].

What is unusual about the present case is the rather late onset at age of 30 , the static type of progression, and the hemihypoesthesia.

\section{Case presentation}

A 35-year-old Dinka woman from South Sudan presented with left-sided facial asymmetry associated with numbness on the left side of her body and deteriorating vision in her left eye. Her symptoms started 5 years earlier with slowly progressive left-sided facial atrophy associated with reduced vision in her left eye and early morning blurred vision. Two years later she started feeling numbness on the left side of her body (upper limb and lower limb spontaneously). Numbness and atrophy became static in the last year with further deterioration in vision.

She never had any seizures or history of a psychiatric illness. There was no family history of a similar condition; she was not on any medication or known to have any chronic illness.

On examination, there was noticeable left-sided facial atrophy extending from the frontal bone to mental region associated with enophthalmos affecting her left eye, minimal tongue atrophy on the same side, and clear coup de sabre in her left cheek (Fig. 1). An intraoral examination revealed minimal tongue atrophy on the left side, badly decayed right maxillary third molar together with the mandibular second and third molars, and there were caries on left mandibular and maxillary third molars.

Her cranial nerves were intact, however, there was decreased sensation (fine touch and pin prick) affecting the left side of her body (including the left side of her face), although tone, reflexes, and proprioception were all intact. An examination of her right side was unremarkable. An ophthalmic examination showed a left eye visual acuity (VA) of 6/40, keratic precipitates, immature cataract, raised intraocular pressure (IOP) of 26, and hazy fundoscopy; however, she had a normal right eye with VA of $6 / 6$ and IOP of 12.2. A brightness scan (B-scan) was performed and it was normal in both eyes (Fig. 2). Other systemic examinations of her heart, chest, and abdomen were unremarkable.

A diagnosis of PRS with overlapped linear scleroderma was postulated and further radiologic and laboratory assessments were made.

General routine investigations revealed normocytic normochromic anemia probably due to amoebiasis infection found in her stool sample (no other agents were seen such as ova or worms) which was corrected after treatment. Liver and renal function tests were within normal ranges as was her vitamin $\mathrm{B}_{12}$ level. Furthermore, rheumatoid factor was positive yet antinuclear antibody (ANA) profile and anti-cyclic citrullinated peptide $(\mathrm{CCP})$ tests were all negative. Laboratory results are shown in Table 1.

Radiological imaging with computed tomography (CT) scan of her facial bones revealed mild asymmetry of her face with early affection of the left zygomatic bone. There was obvious atrophy involving left-sided facial subcutaneous tissue extending to the masseter muscle (Fig. 2). 


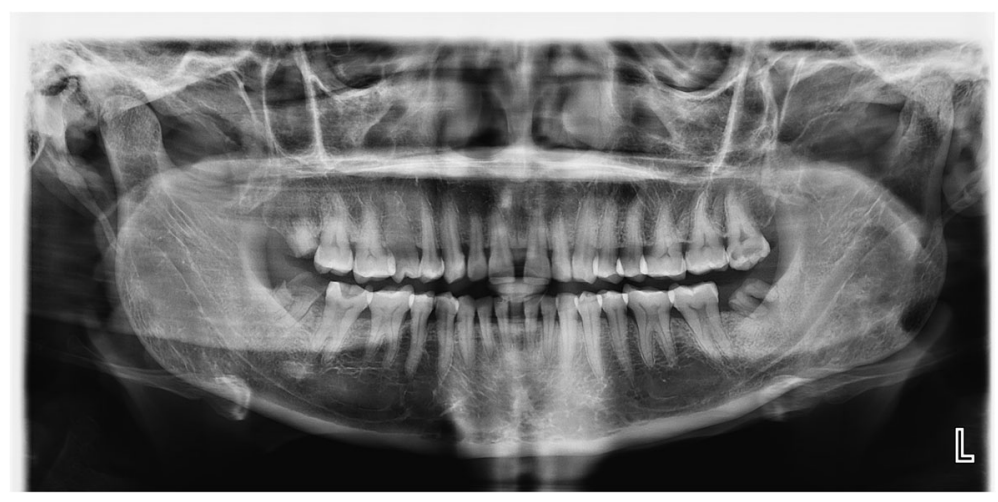

Fig. 3 Orthopantomogram with multiple oral cavities

Moreover, an orthopantomogram (OPG) showed multilocular radiolucency at the left angle of her mandible with remaining roots in the left mandibular third molar, decay in the maxillary left third molar (appeared as radiolucency in the enamel and dentine occlusally), an unerupted right maxillary third molar, and badly decayed mandibular third molar teeth (Fig. 3).

An electroencephalogram (EEG) revealed normal study: normal $9 \mathrm{~Hz}, \alpha$-rhythm in awake EEG with no epileptiform activity seen.

Two skin biopsies were requested that reported: skin with hyperkeratosis, follicular plugging, thin stratum Malpighii, and focal vacuolar degeneration of the basal layer with presence of dermal thick collagen fibers and mononuclear inflammatory cellular infiltration. This picture was consistent with linear scleroderma overlapping PRS.

\section{Discussion}

PRS is a rare neurocutaneous disorder of unknown origin. It is characterized by slowly progressive facial hemiatrophy affecting all tissue layers from skin to bone and frequently (21-31\%) overlaps with a condition known as linear scleroderma "en coup de sabre." It has a prevalence rate of 1 in 70,000 with no difference in rates between different ethnic groups. However, only four cases were reported from Africa in the English language literature: Gueye A, et al. [14], Singh M, et al. [15], Ilhame N, et al. [16], and Elsaid N, et al. [17] from Senegal, Libya, Morocco, and Egypt, respectively. It affects mainly females and usually involves the left side of the face. In most patients $(71 \%)$, disease onset commences before the age of 15 years and only $8 \%$ had an onset after the age of 25 years, as was the case in our patient. Earlier onset and longer duration of PRS have been reported to relate to increased severity of the disease [11, 12, 18-20]

A cerebral disturbance of fat metabolism and atrophic malformation of cervical sympathetic nervous system have been proposed as the primary cause. Other possible factors that are involved in the pathogenesis include trauma, infections (Borrelia burgdorferi and viral), heredity, endocrine disturbances, and autoimmunity [21, 22].

It is associated with different systemic manifestations. It commonly affects the dermatomes of one or multiple branches of the trigeminal nerve with atrophy of the skin and underlying structures (soft tissues, muscles, and bones) and can also affect the eye (enophthalmos, uveitis, retinal vasculitis, glaucoma, central retinal artery occlusion, heterochromic iridocyclitis, restrictive strabismus, Coats disease, papillitis, optic atrophy, and neuroretinitis), the pupil (Horner syndrome, pupillary abnormalities), and hair (band-like alopecia). Also, various oral involvements have been described (unilateral tongue atrophy, jaw hypoplasia, wasting of the muscles of mastication, short roots on affected side, deficiency of soft and hard palate, delayed ipsilateral tooth eruption, and unilateral crossbite). The most commonly seen neurological involvements are epilepsy (60.5\%) followed by pain $(44.2 \%)$; moreover, cerebral vascular malformations have been seen $(11.5 \%)[4,12]$.

Multiple treatment modalities have been mentioned in the literature including:

\section{Medical}

Corticosteroids (topical and intralesional), antioxidants, immunosuppressant (methotrexate), and retinoids.

\section{Surgical}

Fascia grafts, muscle grafts, pedicle flaps, microvascular free flaps, and free fat grafts - injection of aspirated fat and alloplastic graft materials. Some surgical modalities classify the depression type caused by the disease and afterward select the surgical technique to be used [22-24]. 


\section{Conclusion}

PRS is a very rare entity causing progressive hemifacial atrophy that could also be associated with linear scleroderma.

It has devastating outcomes due to its various systemic involvements; therefore, a multidisciplinary approach is required together with further studies to be performed in order to identify the key etiology and construct a clear guideline for management.

\section{Abbreviations}

ANA: Antinuclear antibody; B-scan: Brightness scan; CCP : Cyclic citrullinated peptides; CT: Computed tomographyEEGElectroencephalogram; IOP: Intraocular pressure; OPG: Orthopantomogram; PRS: Parry-Romberg syndrome; VA: Visual acuity

\section{Acknowledgements}

Dr Antony William was consulted for the ophthalmic findings in the case. Dr Vebronia Barsoum was consulted for the dental findings in the case.

\section{Funding}

None declared.

\section{Availability of data and materials}

The authors declare that all the data supporting the findings of this study are available within the article.

\section{Authors' contributions}

JW, took full history and performed full examination. ZT, was consulted about the case and requested investigations needed. RB and YW, majo contributors in writing the manuscript. All authors read and approved the final manuscript.

\section{Authors' information}

Dr Jimmy Girgis William Abdelnour

MBBS, FCCS, MRCP (UK - Part 1), PGCert. DM (Queen Margaret University Edinburgh).

Dr Youeil Girgis William Abdelnour

MBBS, MSc Pediatric Emergency (University of Edinburgh).

Dr Rose-Mery Amin Boushra Kerollos

MBBS

Dr Ziryab Imad Taha Mahmoud

Consultant of Internal Medicine at Haj El-Safi Teaching Hospital, Assistant Professor of Medicine and Head of Department of Internal Medicine at University of Bahri,

MBBS, MRCP (UK), MSc Clinical Pharmacology, Diploma of Research Methodology and Ethics, Rheumatology Fellowship trainee (SMSB).

\section{Ethics approval and consent to participate}

Not applicable.

\section{Consent for publication}

Written informed consent was obtained from the patient for publication of this case report and accompanying images. A copy of the written consent is available for review by the Editor-in-Chief of this journal.

\section{Competing interests}

The authors declare that they have no competing interests.

\section{Publisher's Note}

Springer Nature remains neutral with regard to jurisdictional claims in published maps and institutional affiliations.

\section{Author details}

${ }^{1}$ Abu-Rof Clinic, Omdurman, Sudan. ${ }^{2}$ Khartoum North Teaching Hospital, Khartoum, Sudan. ${ }^{3}$ Haj El-Safi Teaching Hospital, Khartoum, Sudan.
Received: 12 September 2018 Accepted: 1 April 2019

Published online: 03 May 2019

\section{References}

1. Parry $\mathrm{CH}$. Collections from the Unpublished Medical Writings of the Late Caleb Hillier Parry. London: Underwoods; 1825. p. 478-80.

2. Romberg HM. Krankheiten des nervensystems (IV: Trophoneurosen) Klinische Ergebnisse. Berlin: Forrtner; 1846. p. 75-81.

3. Kumar AA, Kumar RA, Shantha GPS, Aloogopinathan G. Progressive hemi facial atrophy - Parry Romberg syndrome presenting as severe facial pain in a young man: a case report. Cases J. 2009;2:6776.

4. Al-Aizari NA, Azzeghaiby SN, Al-Shamiri HM, Darwish S, Tarakji B. Oral manifestations of Parry-Romberg syndrome: A review of literature. Avicenna Jed. 2015;5(2):25-8.

5. $\quad$ Tang XJ, Liu W, Yang B, Shi L, Yin L, Zhang ZY. Parry-Romberg syndrome with rare maxillofacial deformities: A report on two cases. J Craniomaxillofac Surg. 2014;42:780-3.

6. Pensler JM, Murphy GF, Mulliken JB. Clinical and ultrastructural studies of Romberg's hemifacial atrophy. Plast Reconstr Surg. 1990;85:669-74.

7. Ibarra PC. Parry-Romberg syndrome with glaucoma and pregnancy. Gac Med Mex. 2001;137:289.

8. Banks TL, Sugar HS. Ocular manifestations of facial hemiatrophy. J Mt Sinai Hosp N Y. 1963;11:83.

9. El KJ, Abbas O, Rubeiz M. A review of Parry Romberg syndrome. J Dermatol. 2012;67:769-84.

10. Millichap JGA. Neurological Syndromes. New York: Springer; 2013. p. 180.

11. Jun JH, Kim HY, Jung HJ, Lee WJ, Lee S-J, Kim DW, et al. Parry-Romberg Syndrome with en coup de sabre. Ann Dermatol. 2011;23(3):342-7.

12. Stone J. Parry-Romberg syndrome: a global survey of 205 patients using the Internet. Neurology. 2003;61:674-6

13. Badam RK, Dara BB, Pallerla SR, Boringi M. Parry Romberg syndrome: A rare case report. J Indian Acad Oral Med Radiol. 2014;26:237-40.

14. Gueye A, Autret A. First case of facial hemiatrophy (Romberg's disease) observed in Senegal. Bull Soc Med Afr Noire Lang Fr. 1978;23(4):389-91.

15. Singh M, Bharija SC, Belhaj MS, Mangoush E. A: Romberg's Syndrome: A Case Report. Dermatology. 1985;170:145-6.

16. Ilhame N, Christelle E, Mariam M, Karima S, Badredine H. Extra-Cephalic Involvement in the Syndrome of Parry Romberg. Clin Med Img Lib. 2017; 3(3):1-3.

17. Elsaid N, Saied A, El-Salam MA. Egyptian Case of Parry-Romberg Syndrome. American J Rare Dis Diagn Ther. 2018;1(1):001-3.

18. Kumar S, Kumar D, Kumar R, Rajkumar, Prabhakar R. Parry-Romberg Syndrome: A Case of Late Onset with Rapid Progression. J Clin Diagn Res. 2014:8(9):ZD27-8.

19. Sharma M, Bharatha A, Antonyshyn OM, et al. Case 178: Parry-Romberg syndrome. Radiology. 2012;262:721-5.

20. Pichiecchio A, Uggetti C, Egitto MG, et al. Parry-Romberg syndrome with migraine and intracranial aneurysm. Neurology. 2002:59:606-8.

21. Maletic J, Tsirka V, loannides P, Karacostas D, Taskos N. Parry-Romberg Syndrome Associated with Localized Scleroderma. Case Rep Neurol. 2010;2: 57-62.

22. Sargunar B, Ebenezer V, Balakrishnan R, Priya S. Parry Romberg Syndrome A Review of Treatment Options. Biomed Pharmacol J. 2014;7(1).

23. Guerrerosantos J, Guerrerosantos F, Orozco J. Classification and treatment of facial tissue atrophy in Parry-Romberg disease. Aesth Plast Surg. 2007;31: 424-34

24. Anderson LE, Treat JR, Licht DJ, Kreiger PA, Knight AM. Remission of seizures with immunosuppressive therapy in Parry-Romberg syndrome and en coup de sabre linear scleroderma: Case report and brief review of the literature. Pediatr Dermatol. 2018;35:e363-5. 